\title{
Novel diagnostic model for bone metastases in renal cell carcinoma patients based on bone scintigraphy analyzed by computer-aided diagnosis software and bone turnover markers
}

\author{
Takeshi Ujike $^{1}$. Motohide Uemura ${ }^{1} \cdot$ Taigo Kato $^{1} \cdot$ Koji Hatano $^{1}$ - Atsunari Kawashima ${ }^{1}$ - Akira Nagahara ${ }^{1}$. \\ Kazutoshi Fujita $^{1} \cdot$ Ryoichi Imamura $^{1} \cdot$ Norio Nonomura $^{1}$
}

Received: 21 June 2021 / Accepted: 15 December 2021 / Published online: 4 February 2022

(c) The Author(s) 2022

\begin{abstract}
Background Computer-assisted diagnosis (CAD) systems for bone scans have been introduced as clinical quality assurance tools, but few studies have reported on its utility for renal cell carcinoma (RCC) patients. The aim of this study was to assess the diagnostic validity of the CAD system for bone scans and to construct a novel diagnostic system for bone metastases in RCC patients.

Methods We evaluated bone scan images of 300 RCC patients. Artificial neural network (ANN) values, which represent the probability of abnormality, were calculated by BONENAVI, the CAD software for bone scans. By analyzing ANN values, we assessed the diagnostic validity of BONENAVI. Next, we selected 108 patients who underwent measurements of bone turnover markers and assessed the combined diagnostic validity of BONENAVI and bone turnover markers.

Results Forty-three out of 300 RCC patients had bone metastases. The AUC of ANN values was 0.764 and the optimum sensitivity and specificity were 83.7 and $62.7 \%$. By logistic analysis of 108 cases, we found that ICTP, a bone resorption marker, could be a diagnostic marker. The AUC of ICTP was 0.776 and the optimum sensitivity and specificity were 57.1 and $86.8 \%$. Subsequently, we developed a novel diagnostic model based on ANN values and ICTP. Using this model, the AUC was 0.849 and the optimum sensitivity and specificity were 76.2 and $80.7 \%$.

Conclusion By combining the high sensitivity provided by BONENAVI and the high specificity provided by ICTP, we constructed a novel, high-accuracy diagnostic model for bone metastases in RCC patients.
\end{abstract}

Keywords Computer-assisted diagnosis system $\cdot$ Bone scan $\cdot$ Renal cell carcinoma $\cdot$ Bone metastases $\cdot$ Bone turnover marker

\section{Introduction}

Bone metastases are found in approximately $30 \%$ of advanced renal cell carcinoma (RCC) patients, representing the second-most common site of distant metastasis (following lung) [1,2]. Because such lesions profoundly impact quality of life and survival [3], early diagnosis of bone metastases is clinically important. However, there are no firm guidelines for diagnosis of bone metastasis in RCC patients.

Motohide Uemura

uemura@uro.med.osaka-u.ac.jp

1 Department of Urology, Osaka University Graduate School of Medicine, 2-2 Yamadaoka, Suita Osaka 565-0871, Japan
Bone scans are highly sensitive methods for detecting bone metastasis, and are also an effective diagnostic tool for whole-body examinations. Therefore, bone scans are widely used for identifying metastases from various cancers, but it has been reported that they have limitations in detecting osteolytic-type bone metastases, which is the predominant type in RCC [4]. Thus, clinical guidelines for RCC do not recommend bone scans routinely $[5,6]$.

Recently, a computer-assisted diagnosis (CAD) system for bone scans was introduced as a clinical quality assurance tool. There are many reports that the CAD system is useful for both diagnosis and prognostic prediction with respect to prostate cancer exhibiting osteogenic bone metastasis [7-9]. However, there are few reports on the usefulness of RCC exhibiting osteolytic bone metastasis. 
In the diagnosis of bone metastasis of various cancers, many studies on the usefulness of bone turnover markers as a tool for quantitative diagnosis have been made, although this also remains controversial [10-12].

The aim of this study was to assess the diagnostic validity of the CAD system for bone scans and bone turnover markers, and ultimately to develop a novel diagnostic model for bone metastases in RCC patients.

\section{Materials and methods}

\section{Patients characteristics}

We retrospectively selected 300 RCC patients who underwent bone scintigraphy in Osaka University Hospital from January 2008 through May 2016. Every patient was classified into one of two groups - those with and those without bone metastases-based on information from multiple modalities including X-ray, computed tomography (CT), magnetic resonance imaging (MRI), positron emission computed tomography (PET), biopsy result, serial bone scans and other follow-up studies. Using this cohort, we first evaluated the ability of the CAD system to diagnose bone metastases in RCC patients.

Subsequently, we analyzed bone turnover marker data that were available from 104 of the 300 patients. Using this cohort, we examined whether the CAD system and bone turnover markers are useful for diagnosis of bone metastases. Finally, we attempted to construct a novel diagnostic system using factors determined to be informative from logistic regression analysis results.

This study was approved by the Osaka University Hospital institutional review board (The IRB approval number is 12454).

\section{Bone scans}

Patients were injected intravenously with $740 \mathrm{MBq} 99 \mathrm{mTc}$ MDP. At $3 \mathrm{~h}$ after injection, whole-body bone scans were obtained in the anterior view and the posterior view with a gamma camera equipped with low-energy high-resolution parallel hole collimators.

\section{CAD system}

Raw imaging data sets were analyzed by software package BONENAVI version 2 based entirely on a database of individuals in Japan. This CAD system calculates two imaging metrics: one is an artificial neural network (ANN), and the other is bone scan index (BSI). ANN values represented the probability of abnormality. Input factors for ANN calculations were size, shape, intensity, and localization of RI accumulation. An ANN value for each hot spot was calculated by BONENAVI, and this value determined whether a spot was classified as a bone metastasis or not. BONENAVI presented an output between 0 and 1 for each patient as ANN values. ANN values in the range of $0-0.50$ were categorized as benign, while those ranging from 0.51 to 1.00 were categorized as bone metastases. BSI was calculated as the percentage of the sum of all spots classified as bone metastasis in a patient's body. In this study, BSI was not used because the aim of this study was to examine the diagnostic ability of BONENAVI.

\section{Bone turnover markers}

We selected six bone turnover markers: (i) Bone-formation markers, including alkaline phosphatase (ALP) and bonespecific alkaline phosphatase (BAP) and (ii) Bone resorption and osteoclastogenesis markers, including Pyridinoline cross-linked carboxyterminal telopeptide of type I collagen (ICTP), urinary $N$-telopeptide (uNTx), serum $N$-telopeptide (sNTx), and Tartrate-resistant acid phosphatase 5b (TRACP$5 b)$. In addition to these bone turnover markers, we examined eight factors including serum calcium $(\mathrm{Ca})$ and lactate dehydrogenase (LDH).

\section{Statistical analysis}

Results were expressed as the median (range) for continuous variables. Univariate analysis was performed by the Mann-Whitney $U$ test or the chi-square test. Univariate and multivariate logistic regression analyses were performed to determine the correlation between presence of bone metastases and clinical factors (ANN values and bone turnover markers). The predicted probability of bone metastases was estimated as $P=1 /\left(1+\mathrm{e}^{-x}\right)$. Logistic regression yields a score $(X)$, where $X$ is $\beta_{0}+\beta_{1} X_{1}+\beta_{2} X_{2}+\beta_{3} X_{3} \ldots$, which is a linear combination of the predictors $\left(X_{1}, X_{2}\right.$, $\left.X_{3} \ldots\right)$ in the model. The model coefficients $\left(\beta_{0}, \beta_{1}, \beta_{2} \ldots\right)$ were chosen to optimize the ability to predict presence of bone metastases. A nomogram predicting the probability of presence of bone metastases was constructed based on this formula. This new diagnostic model was evaluated for diagnostic ability using the receiver-operator characteristics (ROC) curve analysis. Statistical significance was considered as $p<0.05$. All data analyses were performed with JMP ver.14 (SAS Institute Inc., Cary, NC, USA).

\section{Results}

Patient characteristics are summarized in Table 1. Among 300 patients, 43 patients $(14.3 \%$ ) had metastatic bone tumors. The mean ANN values of the patients with bone 
Table 1 Patients characteristics of all cases

\begin{tabular}{lll}
\hline Variables & \multicolumn{2}{l}{ Metastatic bone tumor } \\
\cline { 2 - 3 } & Negative & Positive \\
\hline Number & 257 & 43 \\
Median age, year (range) & 64 & 68 \\
& $(27-94)$ & $(27-86)$ \\
Gender male/female & $181 / 76$ & $36 / 7$ \\
Tumor histology, $n(\%)$ & & \\
Clear cell & 170 & 23 \\
& $(66.0)$ & $(53.4)$ \\
Papillary & 2 & 2 \\
Chromophobe & $(0.8)$ & $(4.7)$ \\
Spindle & 4 & 2 \\
Unclassified & $(1.6)$ & $(4.7)$ \\
Unknown & 3 & 0 \\
& $(1.2)$ & $(0)$ \\
& 3 & 0 \\
& $(1.2)$ & $(0)$ \\
& 71 & 16 \\
& $(29.2)$ & $(37.2)$ \\
\hline
\end{tabular}

metastases were significantly higher than those without bone metastases $(0.70,0.37$, respectively. $P<0.0001)$ (Fig. 1). The AUCs for the diagnosis of a patient with bone metastasis by BONENAVI were 0.764 and the optimum sensitivity and specificity were 83.7 and $62.7 \%$, respectively (Fig. 2).

Subsequently, we assessed the ability of bone turnover markers to predict bone metastases using clinical data from 104 patients. Patient characteristics are summarized in Table 2. Among 104 patients, 21 patients $(20.2 \%)$ had metastatic bone tumors. In univariate analysis, ICTP of the groups with bone metastases were significantly higher compared with those without bone metastases. The other

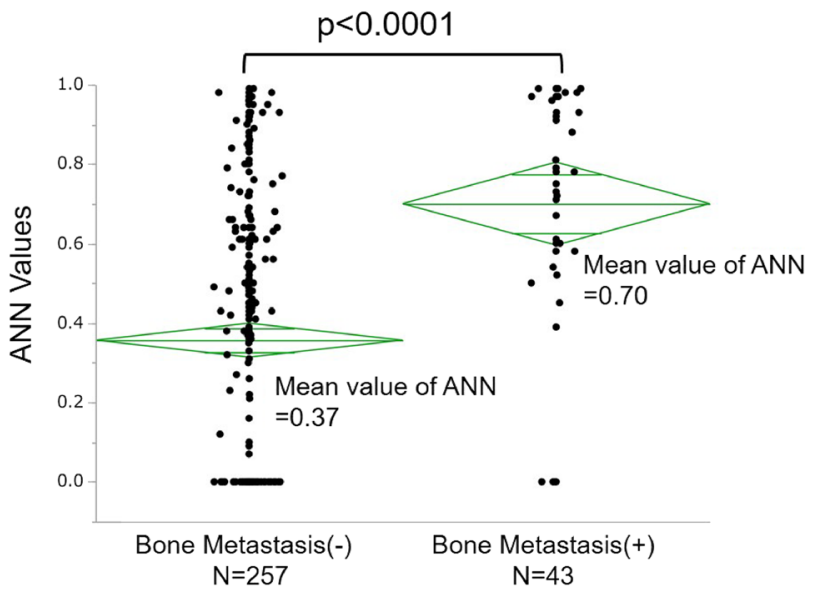

Fig. 1 The plots of the raw data correspond to the true positive of ANNs for RCC patients with bone metastases or without bone metastases

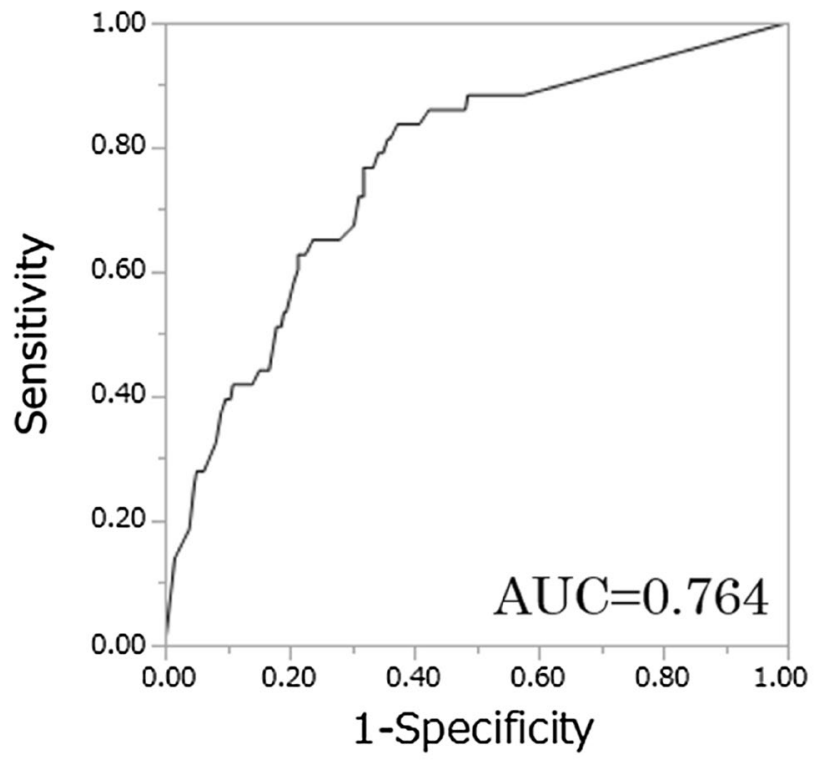

Fig. 2 The receiver operating characteristic (ROC) curves describing the diagnostic performance of BONENAVI to identify bone metastases

factors (BAP, uNTx, sNTX, TRACP5b, ALP, Ca, LDH) were not significantly different between two groups. Multivariate logistic regression analysis revealed that ICTP was an independent predictor for presence of bone metastases $(p<0.0001)$, whereas sNTX were not significant (Table 3$)$. The AUCs for the diagnosis of a patient with bone metastasis by ICTP were 0.776 and the optimum sensitivity and specificity were 57.1 and $86.8 \%$, respectively (Fig. 3A).

Finally, by combining the high sensitivity provided by ANN values and the high specificity provided by ICTP levels, we attempted to construct a novel, high-accuracy diagnostic model for bone metastases in RCC patients. From the result of multivariate logistic regression analysis using ANN values and ICTP levels, we created a novel diagnostic model for the probability of presence of bone metastases as represented by the following formula: $P=1 /\left(1+\mathrm{e}^{-x}\right)$.

$X=3.12-0.890 \times$ ANNscore- $0.230 \times$ ICTP(ICTP: continuous variables, ANN score: ANN $>0.5$ assigns ANN score of $1, \mathrm{ANN} \leqq 0.5$ assigns ANN score of 0 ). Using this diagnostic model (ICTP-ANN model), the AUC for the probability of presence of bone metastases was 0.849 . The optimum sensitivity and specificity were 76.2 and $80.7 \%$, respectively (Fig. 3B).

\section{Discussion}

Identification of bone metastases is essential for making informed treatment decisions for any malignant tumors. Despite the increasing number of reports concerning various 
Table 2 Patient characteristics of 104 patients who were measuring bone turnover markers

\begin{tabular}{|c|c|c|c|c|}
\hline \multirow{2}{*}{\multicolumn{2}{|c|}{ Parameter }} & \multicolumn{2}{|c|}{ Metastatic bone tumor } & \multirow[t]{2}{*}{$P$ value } \\
\hline & & Negative & Positive & \\
\hline \multicolumn{2}{|l|}{ Number } & 83 & 21 & \\
\hline \multicolumn{2}{|l|}{ Median age, year (range) } & $\begin{array}{l}66 \\
(40-94)\end{array}$ & $\begin{array}{l}71 \\
(45-86)\end{array}$ & 0.373 \\
\hline Gender & male/female & $53 / 30$ & $18 / 3$ & 0.055 \\
\hline Stage & I/II/III/IV/unknown & $42 / 4 / 21 / 14 / 2$ & $0 / 1 / 4 / 16 / 0$ & $<0.0001$ \\
\hline \multicolumn{2}{|c|}{$\begin{array}{l}\text { Tumor histology clear cell/papillary/chromophobe/unclassi- } \\
\text { fied/unknown }\end{array}$} & $72 / 0 / 2 / 3 / 6$ & $11 / 3 / 1 / 3 / 3$ & 0.0008 \\
\hline \multicolumn{5}{|c|}{ Median values of bone turnover markers, (range) } \\
\hline ICTP & $(\mathrm{ng} / \mathrm{mL})$ & $\begin{array}{l}4.3 \\
(2.2-15.1)\end{array}$ & $\begin{array}{l}7.2 \\
(2.8-38.1)\end{array}$ & $<0.0001$ \\
\hline BAP & $(\mu \mathrm{g} / \mathrm{L})$ & $\begin{array}{l}12.3 \\
(3.5-51.8)\end{array}$ & $\begin{array}{l}13.5 \\
(7.5-34.4)\end{array}$ & 0.252 \\
\hline uNTx & $(\mathrm{nmolBCE} / \mathrm{mmolCr})$ & $\begin{array}{l}30.0 \\
(5.7-129.6)\end{array}$ & $\begin{array}{l}30.6 \\
(6.9-96.1)\end{array}$ & 0.536 \\
\hline sNTx & (nmolBCE/mmolCr) & $\begin{array}{l}15.6 \\
(8.7-30.0)\end{array}$ & $\begin{array}{l}16.2 \\
(8.6-35.3)\end{array}$ & 0.366 \\
\hline TRACP-5b & $(\mathrm{mIU} / \mathrm{dL})$ & $\begin{array}{l}330 \\
(109-898)\end{array}$ & $\begin{array}{l}353 \\
(87-977)\end{array}$ & 0.606 \\
\hline ALP & (IU/L) & $\begin{array}{l}225 \\
(70-2406)\end{array}$ & $\begin{array}{l}262 \\
(162-1010)\end{array}$ & 0.015 \\
\hline $\mathrm{Ca}$ & $(\mathrm{mg} / \mathrm{dL})$ & $\begin{array}{l}9.2 \\
(8.2-14.6)\end{array}$ & $\begin{array}{l}9.5 \\
(8.4-12.0)\end{array}$ & 0.142 \\
\hline LDH & (IU/L) & $\begin{array}{l}193 \\
(107-532)\end{array}$ & $\begin{array}{l}201 \\
(137-542)\end{array}$ & 0.175 \\
\hline
\end{tabular}

ICTP Pyridinoline cross-linked carboxyterminal telopeptide of type I collagen, $B A P$ bone-specific alkaline phosphatase, $u N T x$ urinary $N$-telopeptide, $s N T x$ serum, $N$-telopeptide, TRACP-5b Tartrate-resistant acid phosphatase $5 \mathrm{~b}, A L P$ alkaline phosphatase, $C a$ serum calcium, $L D H$ lactate dehydrogenase, $P$ value was calculated by Mann-Whitney $U$ test or $\chi$-square test

\begin{tabular}{|c|c|c|c|c|c|c|}
\hline \multirow[t]{2}{*}{ Parameter } & \multicolumn{3}{|c|}{ Univariate } & \multicolumn{3}{|c|}{ Multivariate } \\
\hline & OR & $95 \%$ CI & $P$ value & OR & $95 \%$ CI & $P$ value \\
\hline ICTP & 1.29 & $1.14-1.50$ & $<0.0001$ & 1.31 & $1.14-1.56$ & $<0.0001$ \\
\hline BAP & 1.03 & $0.96-1.10$ & 0.389 & & & \\
\hline uNTx & 1.01 & 0.99-1.04 & 0.328 & & & \\
\hline sNTx & 1.07 & $0.99-1.17$ & 0.093 & 0.95 & $0.84-1.07$ & 0.410 \\
\hline TRACP-5b & 1.00 & $0.99-1.00$ & 0.324 & & & \\
\hline ALP & 1.00 & $0.99-1.00$ & 0.589 & & & \\
\hline $\mathrm{Ca}$ & 1.27 & $0.70-2.22$ & 0.399 & & & \\
\hline LDH & 1.00 & $0.99-1.00$ & 0.267 & & & \\
\hline
\end{tabular}

$O R$ odds ratio, $C I$ confidence interval, ICTP Pyridinoline cross-linked carboxyterminal telopeptide of type I collagen, $B A P$ bone-specific alkaline phosphatase, $u N T x$ urinary, $N$-telopeptide, $s N T x$ serum, $N$-telopeptide, TRACP-5b Tartrate-resistant acid phosphatase 5b, $A L P$ alkaline phosphatase, $C a$ serum calcium, $L D H$ lactate dehydrogenase $P$ value was calculated by Likelihood-ratio test imaging modalities in the diagnosis for bone metastases, the accuracy of each modality, specifically X-ray, CT, MRI, bone scans and PET-CT, still remains unknown and no consensus has been reached. Despite the advent of various imaging modalities, whole-body bone scans remained the standard method for surveying the presence and extent of bone metastasis.

Since bone scans are sensitive in detecting changes in osseous metabolism, it is difficult to distinguish malignant tumors from inflammatory changes and other biological 

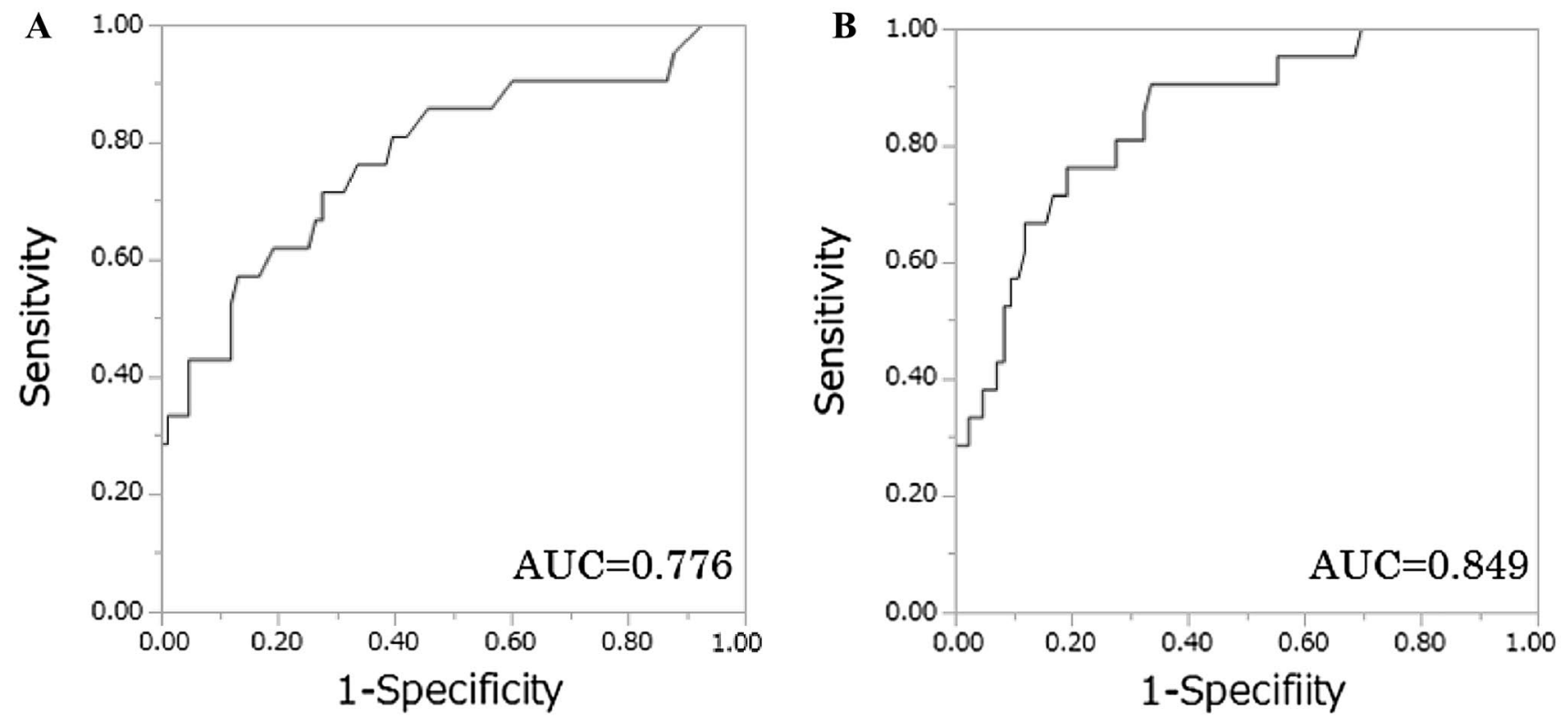

Fig. 3 The receiver operating characteristic (ROC) curves describing the diagnostic performance of ICTP A and novel diagnostic model B to identify bone metastases

phenomena. As a result, bone scans can be prone to false positives. In addition, analysis of bone scans can be highly subjective, making it difficult to obtain diagnoses that are reproducible across multiple clinicians.

To eliminate the need for operator discretion, CAD software, EXINIbone (EXINI Diagnostics AB, Lund, Sweden), for the automated detection and quantification of imaging data, was developed in 2006 [13]. BONENAVI (Fujifilm RI Pharma Co. Ltd., Tokyo), the CAD system adopted in this study, utilizes the same system as EXINIbone. The initial CAD system, EXINIbone, was constructed using a Swedish database. BONENAVI ver.1, was constructed by adopting 904 Japanese patients from a single institution's database [14-16]. BONENAVI ver2, the latest CAD system used in this study, was trained using 1532 Japanese patients from a database comprising nine institutions [17-19]. Diagnostic accuracy using BONENAVI ver2. was superior to those using EXINIbone and BONENAVI ver.1 [17].

BONENAVI generates two imaging markers: an artificial neural network (ANN) value, and a bone scan index (BSI). The ANN value shows the probability of having skeletal metastasis, and the BSI value shows the bone metastatic tumor burden. Many reports have claimed prognosis could be predicted in prostate cancer patients using these two values [9, 20-23].

On the other hand, there are no reports that examined the usefulness of BONENAVI for diagnosis of bone metastasis in RCC patients. Bone metastasis in RCC patients often exhibits osteolytic properties, and it is speculated that it is difficult to detect malignancy by bone scan only. However, we experienced a case where BONENAVI was very useful for diagnosis of bone metastasis (Fig. 4). In this case, BONENAVI clearly confirmed the

\section{A}

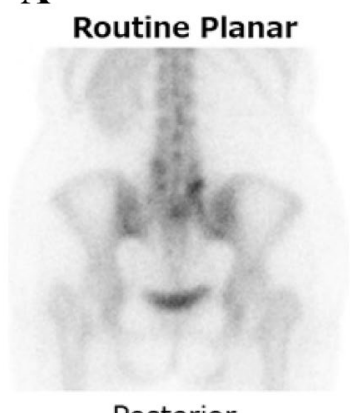

Posterior

B

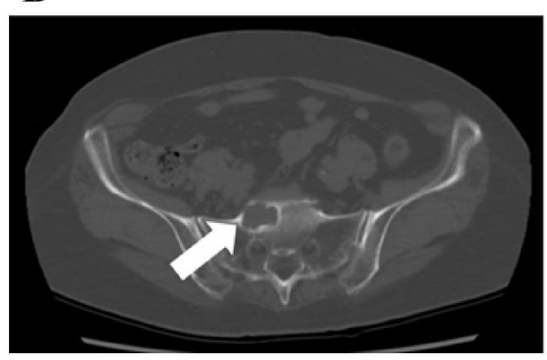

Fig. 4 A 74-year-old female pointed out sacral metastasis nine months after radical nephrectomy. Although RI accumulation of sacral in the routine planar is not clear, it could be clearly judged with BONENAVI. Computed tomography could point out osteolytic changes (white arrow) 
RI accumulation that was difficult to confirm in the routine planar. Finally, a definitive diagnosis was made by histological examination by CT-guided needle biopsy. We hypothesized that BONENAVI could also be useful in diagnosing bone metastases in RCC patients.

In this study, BONENAVI alone had high sensitivity $(83.7 \%)$ on diagnosis for bone metastases, and a certain degree of diagnosis ability was observed. However, the specificity was as low as $62.7 \%$, which was not a satisfactory range. Therefore, to supplement this weak point, we decided to combine the BONENAVI metrics with bone turnover marker measurements, which have been well studied.

For many years, studies have been conducted on various cancers regarding the usefulness of measuring bone turnover markers at the time of diagnosis of bone metastases, but no conclusion has yet been reached [10-12]. On the diagnosis of bone metastases in RCC patients, there are few reports on whether bone turnover markers are useful. Jung et al. assessed the diagnostic accuracy of bone turnover markers in the serum of 72 RCC patients [24]. They examined whether ALP, NTx, TRACP5b, OPG (osteoprotegerin), and RANKL (ligand of the receptor activator of nuclear factor- $\mathrm{\kappa B}$ ) were useful as diagnostic markers for bone metastases. They conclude that bone turnover markers are hardly useful to diagnose bone metastases in RCC patients. However, their study did not assess ICTP.

In this study, we found that ICTP is the most useful among the bone turnover markers we examined. ICTP is a degradation product of type I collagen and is associated with the bone resorption process. It is scientifically consistent that serum ICTP is elevated in RCC patients with bone metastases characterized by osteolytic changes. In the context of lung cancer, reports have claimed that ICTP was higher in patients with bone metastases than those without bone metastases [25]. However, a limited number of cases have been examined, and no conclusion has been reached regarding the usefulness of ICTP.

By combining the high sensitivity provided by ANN values and the high specificity provided by ICTP levels, we were able to construct a novel diagnostic system with high AUC values $(=0.849)$. However, several limitations should be taken into consideration. First, this study consisted of only Japanese patients, and these results may not be applicable to other races. Second, the number of patients who had bone metastasis in this study was relatively small. Third, our study was a retrospective and single-center study. However, we strongly believe that these interesting results can help clinicians treating RCC patients with suspected bone metastases. Of course, to establish the novel diagnostic system of this study, we believe that further large-scale studies are needed.

\section{Conclusion}

We confirmed that the diagnostic ability of BONENAVI for bone metastases in RCC patients is highly sensitive. After confirming this result, we constructed a novel, high-accuracy diagnostic tool for bone metastases in RCC patients by combining the high sensitivity provided by BONENAVI and the high specificity provided by ICTP levels.

\section{Declarations}

Conflict of interest No author has any conflict of interest.

Open Access This article is licensed under a Creative Commons Attribution 4.0 International License, which permits use, sharing, adaptation, distribution and reproduction in any medium or format, as long as you give appropriate credit to the original author(s) and the source, provide a link to the Creative Commons licence, and indicate if changes were made. The images or other third party material in this article are included in the article's Creative Commons licence, unless indicated otherwise in a credit line to the material. If material is not included in the article's Creative Commons licence and your intended use is not permitted by statutory regulation or exceeds the permitted use, you will need to obtain permission directly from the copyright holder. To view a copy of this licence, visit http://creativecommons.org/licenses/by/4.0/.

\section{References}

1. Woodward E, Jagdev S, McParland L et al (2011) Skeletal complications and survival in renal cancer patients with bone metastases. Bone 48:160-166

2. Dabestani S, Thorstenson A, Lindblad P et al (2016) Renal cell carcinoma recurrences and metastases in primary non-metastatic patients: a population-based study. World J Urol 34:1081-1086

3. Santini D, Procopio G, Porta C et al (2013) Natural history of malignant bone disease in renal cancer: final results of an italian bone metastasis survey. PLoS ONE 8:e83026

4. Larcher A, Muttin F, Fossati N et al (2017) When to perform preoperative bone scintigraphy for kidney cancer staging indications for preoperative bone scintigraphy. Urol 110:114-120

5. Ljungberg B, Albiges L, Abu-Ghanem Y et al (2019) European association of urology guidelines on renal cell carcinoma: the update. Eur Urol 75:799-810

6. Motzer RJ, Jonasch E, Agarwal N et al (2017) Kidney cancer, version 2 clinical practice guidelines in oncology. J Natl Compr Canc Netw 15:804-834

7. Dennis ER, Jia XY, Mezheritskiy IS et al (2012) Bone scan index: a quantitative treatment response biomarker for castration-resistant metastatic prostate cancer. J Clin Oncol 30:519-524

8. Ulmert D, Kaboteh R, Fox JJ et al (2012) A novel automated platform for quantifying the extent of skeletal tumour involvement in prostate cancer patients using the bone scan index. Eur Urol 62:78-84

9. Miyoshi Y, Yoneyama S, Kawahara T et al (2016) Prognostic value of the bone scan index using a computer-aided diagnosis system for bone scans in hormone-naive prostate cancer patients with bone metastases. BMC Cancer 16:1-9 
10. D’Oronzo S, Brown J, Coleman R (2017) The role of biomarkers in the management of bone-homing malignancies. J Bone Oncol 9:1-9

11. Alcaraz A, Gonzalez-Lopez R, Morote J et al (2013) Biochemical markers of bone turnover and clinical outcome in patients with renal cell and bladder carcinoma with bone metastases following treatment with zoledronic acid: the TUGAMO study. Br J Cancer 109:121-130

12. Yao S, Zhang YL, Tang L et al (2017) Bone remodeling and regulating biomarkers in women at the time of breast cancer diagnosis. Breast Cancer Res Treat 161:501-513

13. Sadik M, Jakobsson D, Olofsson F et al (2006) A new computerbased decision-support system for the interpretation of bone scans. Nucl Med Commun 27:417-423

14. Kikuchi A, Onoguchi M, Horikoshi H et al (2012) Automated segmentation of the skeleton in whole-body bone scans: influence of difference in atlas. Nucl Med Commun 33:947-953

15. Takahashi Y, Yoshimura M, Suzuki K et al (2012) Assessment of bone scans in advanced prostate carcinoma using fully automated and semi-automated bone scan index methods. Ann Nucl Med 26:586-593

16. Tokuda O, Harada Y, Ohishi Y et al (2014) Investigation of computer-aided diagnosis system for bone scans: a retrospective analysis in 406 patients. Ann Nucl Med 28:329-339

17. Nakajima K, Nakajima Y, Horikoshi H et al (2013) Enhanced diagnostic accuracy for quantitative bone scan using an artificial neural network system: a Japanese multi-center database project. EJNMMI Res 3:1-9

18. Koizumi M, Wagatsuma K, Miyaji N et al (2015) Evaluation of a computer-assisted diagnosis system, BONENAVI version 2, for bone scintigraphy in cancer patients in a routine clinical setting. Ann Nucl Med 29:138-148
19. Kikushima S, Hanawa N, Kotake F (2015) Diagnostic performance of bone scintigraphy analyzed by three artificial neural network systems. Ann Nucl Med 29:125-131

20. Mitsui Y, Shiina H, Yamamoto Y et al (2012) Prediction of survival benefit using an automated bone scan index in patients with castration-resistant prostate cancer. BJU Int 110:E628-E634

21. Uemura K, Miyoshi Y, Kawahara T et al (2016) Prognostic value of a computer-aided diagnosis system involving bone scans among men treated with docetaxel for metastatic castration-resistant prostate cancer. BMC Cancer 16:1-9

22. Inaki A, Nakajima K, Wakabayashi H et al (2019) Fully automated analysis for bone scintigraphy with artificial neural network: usefulness of bone scan index (BSI) in breast cancer. Annals nucl med 33(10):755-765

23. Ito I, Ito K, Takahashi $\mathrm{S}$ et al (2017) Association between bone scan index and activities of daily living in patients with advanced non-small cell lung cancer. Support Care Cancer 25:1779-1785

24. Jung K, Lein M, Ringsdorf M et al (2006) Diagnostic and prognostic validity of serum bone turnover markers in metastatic renal cell carcinoma. J Urol 176:1326-1331

25. Kong QQ, Sun TW, Dou QY et al (2007) beta-CTX and CTP act as indicators of skeletal metastasis status in mace patients with non-small cell bung cancer. Int J Biol Markers 22:214-220

Publisher's Note Springer Nature remains neutral with regard to jurisdictional claims in published maps and institutional affiliations. 